\title{
Early cystic thoracic cord cavity complicating decompressive surgery for spondylotic spinal canal stenosis. Case report
}

\author{
JC García-Moncó ${ }^{1}$, MG Beldarrain ${ }^{1}$, MJ Ereño ${ }^{3}$ and A Alvarez ${ }^{2}$ \\ Section of ${ }^{1}$ Neurology and ${ }^{2}$ Traumatology, Hospital de Galdacano, 48960 Galdacano, Vizcaya, Spain; ${ }^{3}$ Centro \\ de Resonancia Magnetica Bilbao, Clínica San Francisco Javier, Bilbao, Vizcaya, Spain
}

This is a report of a patient who developed thoracic cystic myelopathy immediately following an otherwise successful decompression operation for spondylotic stenosis secondary to osteophyte formation of the thoracic spinal canal. As this case shows, thoracic laminectomy is not without risks, and cystic myelopathy is a potential complication that should be borne in mind when surgery for spondylosis is being planned. Cavitation of the spinal cord may be associated with trauma secondary to extramedullary cord compression.

Keywords: thoracic spinal cord; spinal cord decompression; osteophyte; cystic myelopathy; paraplegia; postoperative complications

\section{Case report}

A 48-year-old man was admitted to our hospital with a history of several months of back pain and associated walking difficulties in the previous few weeks. The pain was dull, ill-defined, with no radicular characteristics and he denied sphincter disturbances or impotence. Physical examination revealed mild spasticity and hyperactive deep tendon reflexes in both legs, right more so than left, with normal tone and normal reflexes in both arms. The plantar responses were equivocal. Sensory examination was unremarkable except for mild bilateral proprioceptive loss distally in lower limbs. A CT scan revealed an osteophyte compressing the spinal cord posteriorly at T10-11 level. Magnetic resonance imaging (MRI) (Figure 1 and 2) confirmed the finding and showed no evidence of compression at any other level. At laminectomy the surgeon noticed dural impression at the level of the stenosis. A laminectomy was carried out and the stenosis relieved. No trauma to the spinal cord was thought to have occurred; the dura was not opened during surgery. Pathological examination was compatible with an osteophyte rather than with a calcified ligamentum flavum. The patient developed immediate paraplegia and urinary retention. There was hyperreflexia, bilateral Babinski signs and a normal sensory examination except for the previously mentioned findings of distal proprioceptive loss. MRI (Figure 3) performed 3 days after surgery showed a cystic cavity of some $1.5 \mathrm{~cm}$ in length at the T10-11 level, with no evidence of spinal canal stenosis. This was not apparent on the previous study. In the following weeks, the patient complained of impotence. After rehabilitation, $1 \frac{1}{2}$ years after surgery, the patient has been left with sexual dysfunction and marked lower limb spasticity and hyperreflexia, requiring two supports when walking.

\section{Discussion}

In this patient, both long term spinal cord compression by the osteophyte and the surgery could have contributed to the development of the cavity. As far as we know, there was no surgical trauma. Immediately after surgery there was worsening of symptoms and signs that were found preoperatively; and there was also impotence. This suggests that previous, chronic trauma or ischaemia may have played a role in the pathogenesis. Middleton et $\mathrm{al}^{1}$ have described a patient with cervical spondylotic myelopathy who developed a cervical syrinx and neurological deterioration in the immediate postoperative period following decompressive laminectomy. This complication may be more common than has been previously recognised and has been discussed by Dohrmann and Rubin ${ }^{2}$ as a possible factor contributing to suboptimal clinical results in patients undergoing treatment for cervical spondylosis. Syringomyelia should not be diagnosed unless at least two spinal cord segments are involved; therefore, in our patient cystic myelopathy appears to be a more appropriate term than syringomyelia.

It has been proposed that long-standing compression of the spinal cord due to an extramedullary mass may lead to neuroglial damage, which is manifested by enlargement of the extracellular perivascular spaces; ${ }^{3}$ this may have been a factor in this patient, since the small cavity was located at the level of the cord compression, and the symptoms appeared early and were similar to those present before the operation.

Another important point is that spinal cord cavities may cause symptoms and signs that are difficult to differentiate from spondylosis, based on the clinical history and the physical examination. Also, coincidental asymptomatic spondylosis is a frequent finding even at a young age. The advent of MRI has allowed us to recognise cystic myelopathy more frequently, and perhaps this disorder has often been missed in patients previously operated on for spinal canal disorders.

The possibility of cystic myelopathy complicating surgery for spondylosis of the spinal canal should be considered whenever early clinical deterioration 
จ

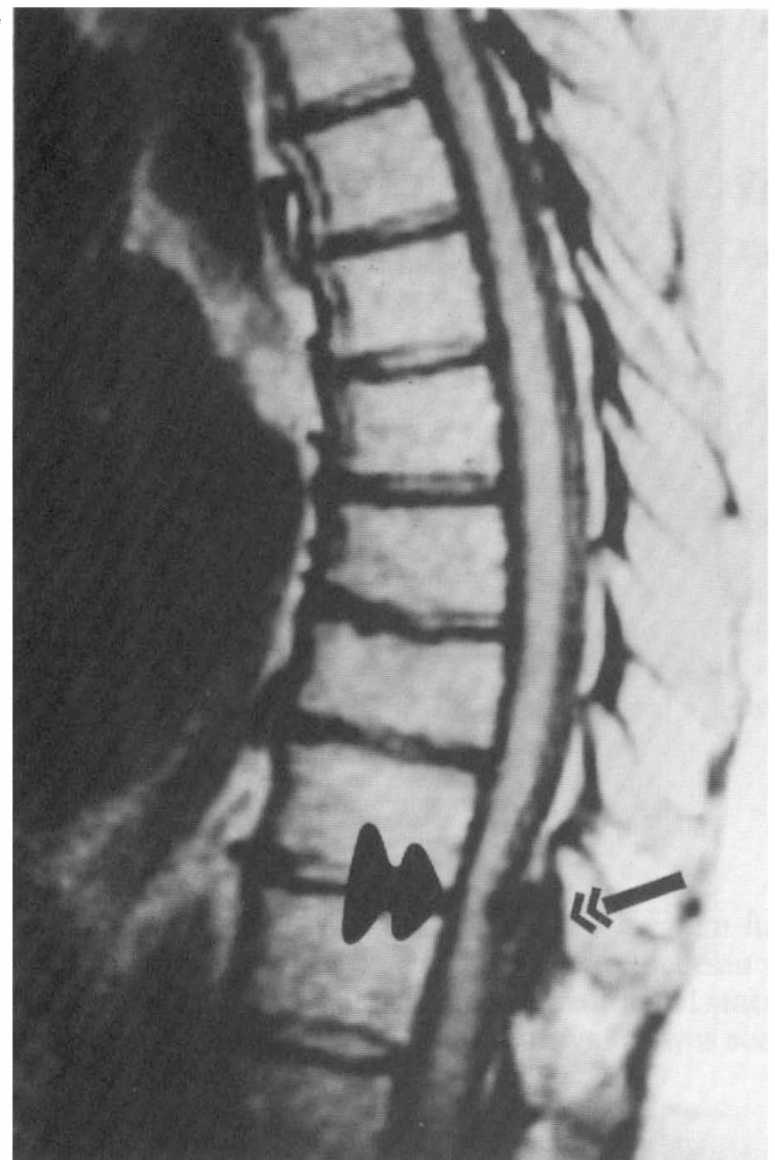

b

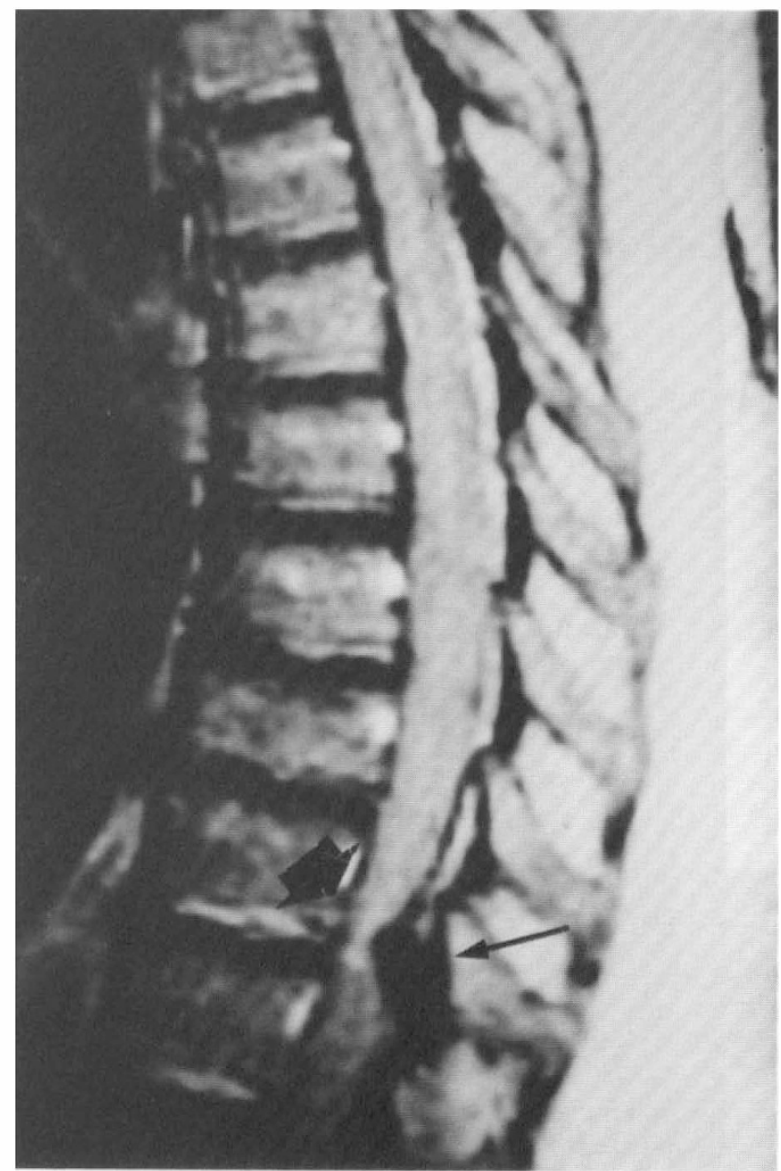

Figure 1 Sagittal image of the thoracic spine (T10-11 level) before surgery. (a) Proton-density image (TR $1300 \mathrm{~ms} / \mathrm{TE}$ $25 \mathrm{Ms}$ ). (b) T2-weighted SE sequence (TR $1300 \mathrm{~ms} / \mathrm{TE} 80 \mathrm{~ms}$ ). Focal hypointensity dorsal to the spinal cord representing ossification of the ligamentum flavum, which contributes to posterior encroachment upon the spinal cord (thin arrow)

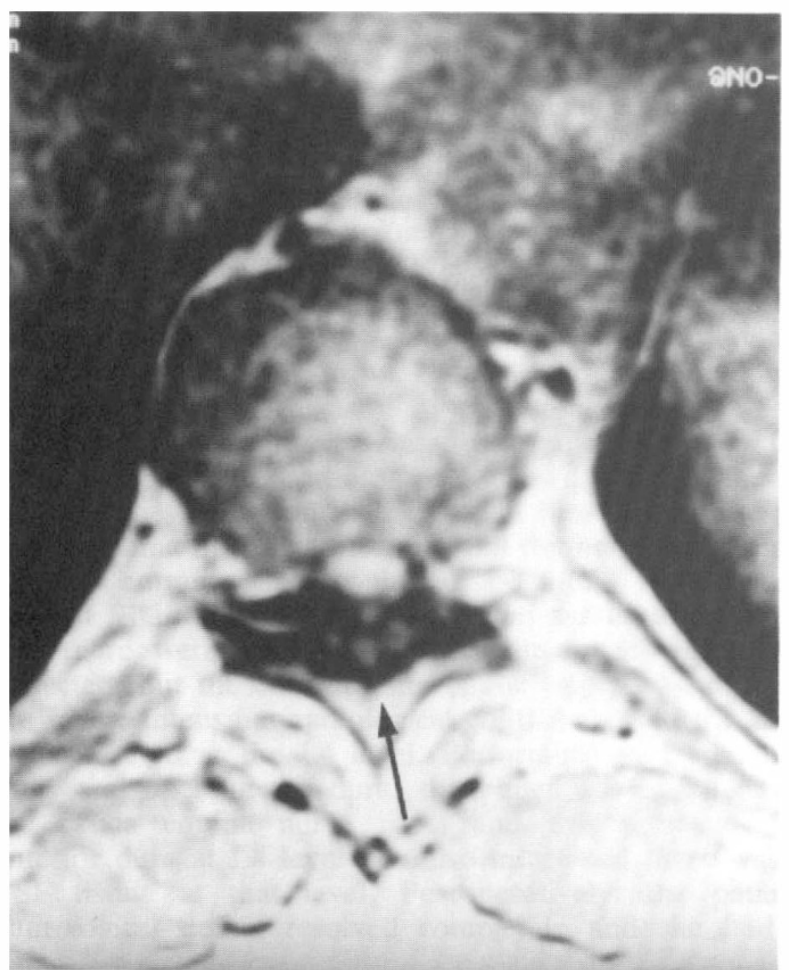

Figure 2 Axial gradient-echo technique (TR 380/TE 15; $100^{-}$) image of the thoracic spine (T10-11 level) before surgery. The area of low signal intensity dorsal to the thecal sac is well demonstrated here (arrow) representing severe ligamentum flavum thickening and osteophyte formation 
540

a

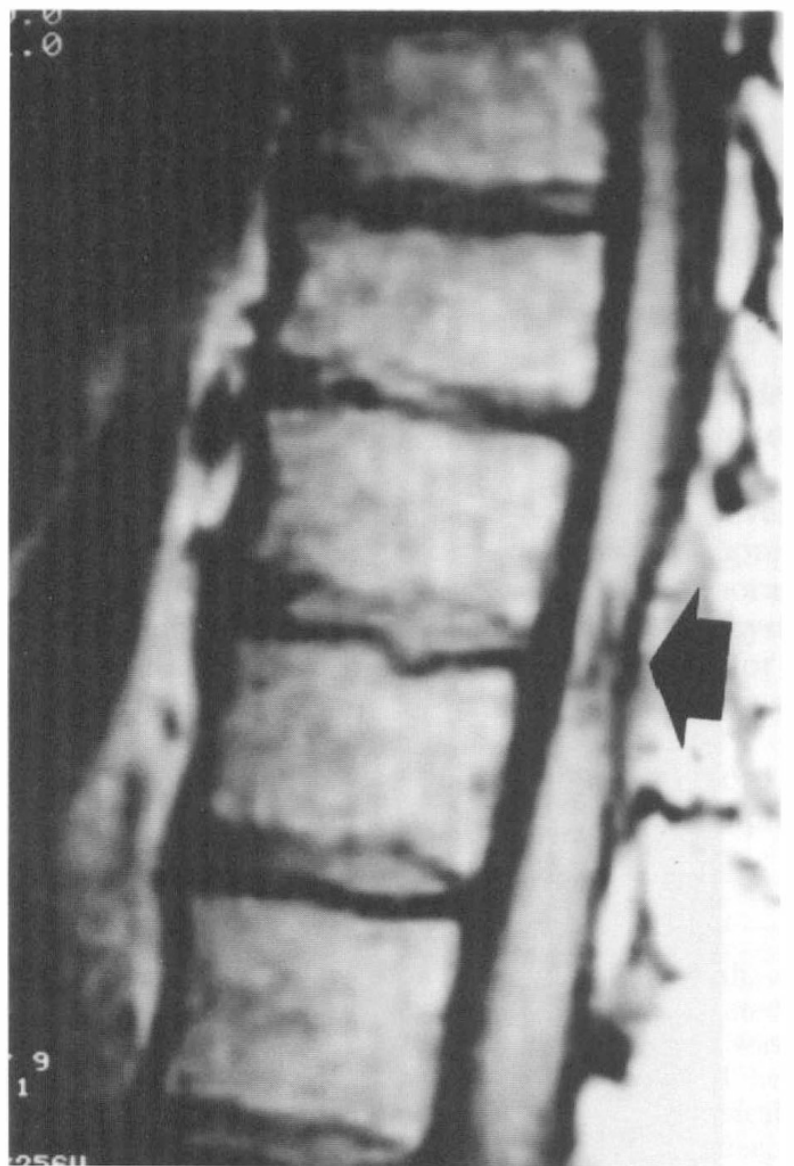

b

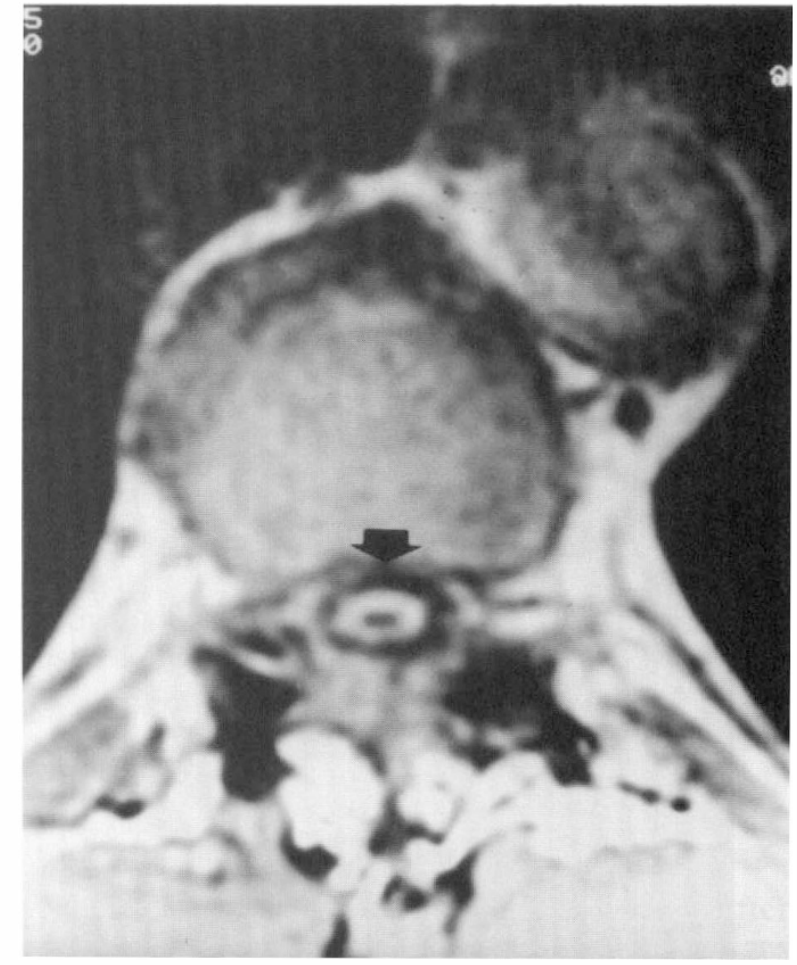

Figure 3 Postoperative images. (a) Sagittal (SE 560/25) showing well defined area of hypointensity within the cord at the level of the previous compression. (b) Axial gradient-echo technique (GE 380/15; 100ํำ). Restoration of the normal contour of the cord with an area of decreased signal (arrow) that parallels the intensity of CSF

occurs, and MRI is the diagnostic method of choice for its detection. Thoracic laminectomy for compressive lesions may be exceptionally hazardous. To reduce the risk of surgical trauma, the use of a high speed drill and the avoidance of footed instrumentation is of paramount importance in removing posterior compressive lesions, and anterior lesions should always be approached by an anterior operative exposure.

\section{References}

1 Middleton TH et al. Syringomyelia after decompressive laminectomy for cervical spondylosis. Surg Neurol 1987; 28: 458-462.

2 Dohrmann GJ, Rubin JM. Cervical spondylosis and syringomyelia: suboptimal results, incomplete treatment, and the role of intraoperative ultrasound. Clin Neurosurg 1986; 34: 378-388.

3 Quencer RM, EI Gammal T, Cohen G. Syringomyelia associated with intradural extramedullary masses of the spinal canal. $A J N R$ 1986; 7 : 143-148. 